\title{
Informe de primera etapa del proyecto de investigación sobre el tema "La modificación de los hábitos y rutinas diarias de personas afectadas por la catástrofe hídrica de la ciudad de Santa Fe"
}

\author{
Boggio R., Carla ${ }^{[1]}$; Chiapessoni, Daniela ${ }^{[2]}$; Funes, Juan Carlos ${ }^{[3]}$; del Valle Arenaza, María ${ }^{[4]}$; Boffelli, \\ Mariana ${ }^{[5]}$; Heit, Mariquita ${ }^{[6]}$; Demichelis, Mauro ${ }^{[7]}$; Demiryi, Milagros ${ }^{[8]}$
}

\section{- Resumen}

En Abril del 2003, la ciudad de Santa Fe (Argentina) vivió la más dramática de las catástrofes en la historia provincial y nacional. El río Salado desbordo junto a la impericia, imprevisión e irresponsabilidad de los gobernantes afectando directamente a 150.000 habitantes. Producto de esto, a las pérdidas en vidas, viviendas, instalaciones y producción, hay que sumar los daños en salud, educación, fuentes laborales e identidad de las personas, familias, barrios y comunidades, que perduran en el tiempo.

Los cambios producidos en las ocupaciones y las representaciones de personas, han generado nuevas configuraciones cuya dimensión ocupacional se desconoce.

Las ocupaciones caracterizan la vida humana, constituyen fuente de identidad personal y social, organizan la vida cotidiana aportando a los estados de salud y función ocupacional. Una de sus expresiones es a través de las rutinas y hábitos cotidianos.

Desde la Universidad Nacional del Litoral, un grupo de terapeutas ocupacionales conformamos un equipo de investigación con la expectativa de producir conocimientos que permitan caracterizar el impacto que la catástrofe ha producido en la dimensión ocupacional de las personas.

El diseño adopta metodología cualitativa para captar el fenómeno a profundidad y desde la perspectiva de los afectados.

Los propósitos que se pretenderan alcanzar con el desarrollo del trabajo, son:

1. Analizar: a) los cambios de rutinas y hábitos con mayor impacto en la vida de las personas; b) las características de los procesos de interrupción y adaptación ocupacional. 2. Construir indicadores de necesidades ocupacionales desde la perspectiva de los afectados. 3. Detectar los soportes más efectivos para la recuperación ocupacional.

Palabras claves: catástrofe, hábitos, rutinas, terapia ocupacional. 


\begin{abstract}
- Abstract
In April $27^{\text {th }}$ 2003, Santa Fe, Argentina, underwent the most dramatic catastrophe in the provincial and national history. The Salado River overflowed due to the unskillfulness, lack of foresight and irresponsibility of the politicians, affecting 150.000 inhabitants. This resulted in loss of lives, housing, installations and equipments, not to mention the damage in health, education, sources of labour, personal identity, families, neighbourhoods and communities, which are everlasting.
\end{abstract}

This has changed life, occupation and representation of people, giving origin to new configurations, the occupational dimensions of which are unknown.

The human life is characterized by the occupations which constitute sources of personal and social identity, and organize the daily life contributing with health and occupational function states. One of their expressions is through everyday routines and habits.

A study was initiated in the National University of Litoral in order to know the changes in routine life habits caused in the occupational dimension, and the adaptation processes initiated by the victims.

Qualitative methods were employed to collect data to understand the phenomenon and to obtain information for the perspective of the affected people.

The pursued objectives include:

1. To identify and analyze the changes in routines and habits with major impact in people's life. 2. To identify and establish necessity indicators from the perspective of the affected people.3. To detect the most effective supports for the occupational recovery.

Key words: catastrophe, habits, routines.

[1], [2], [3], [4], [5]Terapista Ocupacional. ${ }^{[6]}$ Licenciada en Terapia Ocupacional 


\section{Introducción}

El presente trabajo constituye el primer esfuerzo sistematizado en la Argentina, por desarrollar y validar conocimientos e instrumentos de la disciplina, relacionados con el fenómeno de las catástrofes. Cuenta con la acreditación de la Universidad Nacional del Litoral, formando parte de los Proyectos de Investigación y Desarrollo 2005 de la Secretaría de Ciencia y Técnica.

Surge por iniciativa de un grupo de terapeutas ocupacionales que detecta vacíos críticos en la experiencia de intervención directa durante la catástrofe hídrica producida en Santa Fe desde la inundación del 2003 y en las etapas posteriores, que se extienden hasta la actualidad. Este proyecto cuenta con la aprobación por parte de organismos calificados. Se desarrollara durante tres años, obteniéndose conclusiones sobre los interrogantes del equipo, en el año 2008, fecha que finaliza. Actualmente esta cursando el primer tramo que corresponde a la revisión bibliográfica relacionada con el tema, los protocolos de intervención que existen y la definición de aspectos relevantes sobre los instrumentos de recolección de datos. La inquietud del equipo es compartir los avances del trabajo hasta aquí obtenidos.

Esta dolorosa experiencia ha puesto en evidencia la crisis de ciertos conocimientos académicos y la falta de formación específica en el tema, en la mayoría de las disciplinas, ya que entendemos que las ciencias y sus respectivas disciplinas cumplen con funciones cognitivas pero también políticas.

\section{-Antecedentes "}

Hasta la década del 60, las áreas privilegiadas de investigación en relación a los fenómenos de catástrofes fueron las ciencias físicas y la ingeniería, configurando la visión dominante sobre la problemática que Henitt (1983), caracterizó como enfoque tecnocrático. Hasta ese momento, las ciencias sociales ocuparon un rol secundario en el análisis del tema. $(\underline{1})$

De allí en más, surge una línea de investigación que plantea una visión alternativa al "paradigma estructural" (Smith, 1996). Esta línea procura hacer visibles la dimensión social de las situaciones de catástrofes. Visión que ha sido desarrollada por psicólogos comunitarios, sociólogos, antropólogos, geógrafos, entre otras disciplinas, todos con considerable experiencia en países del tercer mundo(르.

Se incorpora así la noción de riesgo que integra la visión fisicalista y el enfoque social. Los modelos holísticos conceptualmente entienden el riesgo como una construcción dinámica dada por la interacción entre amenazas, vulnerabilidad, pérdidas, daños y estrategias de adaptación. El daño como evidencia empírica materializa el riesgo(3).

Los aportes de autores como Gilbert White, dentro de la perspectiva de la ecología humana, o de Westgate, Wisner, O'Keee, Hewwitt y otros, desde la perspectiva de la economía política, encontraron un fuerte eco en los años $1990^{(4)}$.

En 1992, la creación de la Red de Estudios Sociales en Prevención de Desastres en América Latina buscaba fortalecer las visiones sociales sobre los desastres y 
establecer un más justo equilibrio entre los aportes necesarios de las ciencias básicas y las ciencias sociales ${ }^{(\underline{5})}$.

Por su parte el sector salud, con fuerte gravitación en el tema, ha producido abundantes conocimientos en relación al impacto de las catástrofes en los aspectos psicofísicos de la salud de los damnificados ${ }^{(\underline{6})}$. Conocimientos que han avanzado del enfoque clínico con tipificaciones tales como el PSTD (Post Traumatic Stress Disorder, $D S M I V)$, a los enfoques epidemiológico y comunitario, que enfatizan en estrategias de atención primaria de la salud de las víctimas de desastres ${ }^{(\underline{7})}$.

Los avances en el conocimiento del tema permiten afirmar la idea de que un solo gran desastre es en realidad un número infinito de pequeños desastres que afectan de manera diferenciada a múltiples individuos, familias, comunidades y zonas. Resultando éste el ámbito más apropiado para describir y captar la dimensión del impacto desde su expresión en daños y pérdidas ${ }^{(\underline{8})}$.

La Organización Panamericana de la Salud (OPS) y la Organización Mundial de la Salud (OMS), como organismos de indiscutible trayectoria, han realizado aportes de manera sistemática y permanente desde programas específicos sobre el tema.

Hay antecedentes internacionales de estudios de la disciplina sobre efectos de catástrofes, aunque no son específicos del tema que se propone estudiar este trabajo. Entre ellos, el de Rosenfeld, M. S. (1987), que examinó la interrupción de las rutinas de vida cotidiana causados por incendios domésticos y de los procesos de adaptación que emprenden las víctimas para reestablecer patrones eficaces de actividad dirigida. $\underline{(\underline{9}-\underline{10})}$

No se han encontrado investigaciones en terapia ocupacional a nivel internacional y local sobre las rutinas de personas afectadas por inundaciones.

Se identificaron antecedentes de investigaciones en T.O. sobre las rutinas cotidianas de personas con afecciones patológicas. Entre ellos la de Bridle. M. J., Link, B. Y Quessembery, C.M. (1990), que incluyó a adultos con lesión medular en las áreas de organización de rutinas de la vida cotidiana y la de Weeder, T. (1986), que comparó a adultos esquizofrénicos con adultos normales en los patrones temporales de las actividades cotidianas y el significado de esas actividades cotidianas. $\underline{(11-\underline{12})}$

A las referencias internacionales citadas se puede agregar la investigación realizada en Argentina sobre las consecuencias psicológicas de las explosiones ocurridas en la Fábrica Militar de Río III, Córdoba Los autores advierten sobre la necesidad de actualizar los conocimientos sobre hechos catastróficos para fortalecer las redes de prevención, mitigación y rehabilitación. ${ }^{(13)}$.

En el plano local, las recientes investigaciones de Algarañas, Badarioti y Bossio (2003) en relación a la "Organización de la rutina de personas afectadas por el Mal de Alzheimer en el estadío I de la enfermedad que concurren al consultorio externo del Hospital J. B. Iturraspe de la ciudad de Santa Fe" y la de Heinrich, Negri, Ruiz (2004) sobre "La rutina diaria de ancianos institucionalizados en geriátricos de la ciudad de Santa Fe", dan cuenta de los avances del conocimiento disciplinar en las características de las rutinas diarias en poblaciones específicas, a la vez que ponen en evidencia que sigue siendo un área sin investigar aún las consecuencias de la catástrofe hídrica desde la perspectiva ocupacional. $\left.{ }^{(14}\right)$ 
En nuestra ciudad, desde diversas disciplinas se han emprendido estudios sobre la catástrofe hídrica pero no existen antecedentes nacionales ni locales de investigaciones en terapia ocupacional.

A partir de este estado del arte, se puede afirmar que la dimensión ocupacional del impacto de la catástrofe es aún un tema desconocido y de vacancia. La vigencia del tema se actualiza dramáticamente a la luz de los acontecimientos catastróficos sucedidos en el mundo en los últimos tiempos.

El presente trabajo constituye el primer esfuerzo sistematizado en la Argentina, por desarrollar y validar conocimientos e instrumentos de la disciplina con este recorte temático. Cuenta con la acreditación de la Universidad Nacional del Litoral, formando parte de los Proyectos de Investigación y Desarrollo 2005 de la Secretaría de Ciencia y Técnica.

\section{Objetivos generales que se pretenden alcanzar en el periodo total del proyecto}

- Contribuir al proceso de re-construcción post-catástrofe de la vida de las personas, familias y grupos de la comunidad de Santa Fe.

- Aportar al mejoramiento de la calidad de vida de las personas afectadas por la catástrofe hídrica.

- Contribuir al debate sobre y en relación a catástrofe desde la perspectiva de la Terapia Ocupacional y las Ciencia de la Ocupación.

\section{Objetivos generales que tenderán al logro de objetivos generales}

- Identificar y analizar los cambios de hábitos y rutinas que mayor impacto han producido en la vida de las personas.

- Analizar y ponderar las características de los procesos de interrupción y adaptación ocupacional.

- Identificar y construir indicadores de necesidades desde la perspectiva de los afectados y las categorías de análisis de la disciplina.

- Detectar los soportes que resultan más efectivos para la recuperación funcional.

- Elaborar una propuesta curricular que incorpora el tema catástrofe en los planes de estudio de las carreras de la Escuela Superior de Sanidad (Licenciatura en Terapia Ocupacional, Licenciatura en Saneamiento Ambiental y Licenciatura en Administración Hospitalaria).

\section{Metodología}

Se utilizará metodología cualitativa, ya que condice con los intereses de la terapia ocupacional en general y con los objetivos de este trabajo en particular, por centrarse ambas en las realidades de la vida diaria, y poner énfasis en el conocimiento y comprensión de los cambios producidos en las rutinas desde la perspectiva de los afectados.

La selección de los participantes se realizará acorde a criterios no probabilísticos, definiendo la muestra por conveniencia. 
Las unidades de análisis serán personas adultas de diferente sexo, ocupación, que hayan sido afectadas directamente por la inundación, acepten participar en el estudio y den constancia de ello; pertenezcan a diferentes grupos etarios y socioculturales, y que estén en condiciones de hablar de su propia vivencia. Se realizara una pesquisa naturalista en el ambiente propio de los participantes.

Para la recolección de datos se pretenderá implementar entrevistas en profundidad , previa capacitación y entrenamiento de los investigadores para el uso del instrumento captando la información desde la perspectiva de los informantes. Se reconstruirán los campos temáticos en relación al problema de investigación: "Modificaciones en los hábitos y rutinas diarias" en tres momentos temporales diferentes: antes de la inundación, durante la inundación y después de la inundación. A modo de captar la línea de los cambios.

La construcción de instrumentos se realizará en base a los constructos teóricos y marcos de referencia del Modelo de la Ocupación Humana.

Desde la Ciencia de la Ocupación resulta también pertinente para la comprensión de este fenómeno, los aportes de Yerxa, E. (1989), sobre el ser ocupacional; de Clark F. (2000), sobre hábitos y rutinas ocupacionales y el tiempo como fuerza importante para la organización ocupacional; y los de Hocking y Levine (1997) sobre las nociones de temporalidad y los procesos ocupacionales. $\underline{(15}-\underline{16})$

Se considera pertinente la combinación de la utilización de historias de vida y la narrativa ocupacional, por la naturaleza del tema. Metodológicamente, tomar la perspectiva de los directos afectados permitirá captar una información que no está disponible y constituirá un aporte tanto para la evidencia empírica como para los constructor teóricos. La narrativa y el significado que las personas otorguen a las vivencias (Polkinghorne, D. 1996); los eventos escogidos, las descripciones, el orden, la secuencia, abrirán la puerta a la comprensión para producir intervenciones que aporten al bienestar de las personas y las comunidades. La casa en donde se vive, el trabajo, el estudio, los objetos personales y laborales, el barrio, los vecinos; se revisten de sentido según las experiencias, metas, sueños, proyectos y oportunidades que cada cual tenga individual y colectivamente. La fuerza de la narrativa permitirá comprender la identidad, proveerá dirección y propósito al unir eventos de la vida a través del tiempo. $(\underline{17})$

Se aplicará el análisis de contenido que permitirá establecer categorías para codificar los datos. Las categorías incluirán tipos de cambios en los hábitos y rutinas diarias, áreas y contextos de desempeño ocupacional y patrones de habituación. Así también como tipos de necesidades según los momentos temporales y tipos de apoyos efectivos.

La triangulación en el proceso de análisis de datos se implementará con el propósito de elevar la validez de los conocimientos producidos.

\section{Expectativas}

Se espera lograr la producción de conocimientos que permitan caracterizar el impacto que la catástrofe ha producido en la dimensión ocupacional de la vida de las personas. 
Los resultados se constituirán en insumos básicos para el sistema de salud en general, para las políticas sociales y para las organizaciones de la sociedad civil.

Se aspira a contribuir con la formación de recursos en el área de investigación de terapia ocupacional (para alumnos y docentes) y con la producción de aportes curriculares que cualifiquen la formación de los futuros profesionales del área de salud en general y de Terapia ocupacional, en particular.

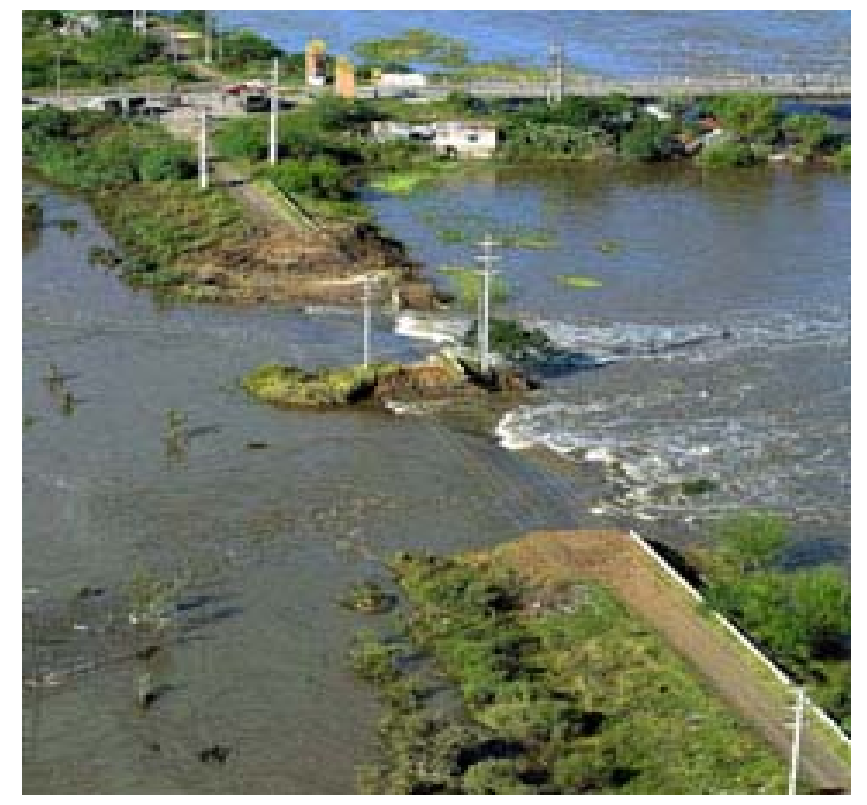

"... no es posible olvidar que detrás del avance del agua había personas a las que la inundación cambió sus vidas, acaso, para siempre..."

Mariano Nimo. Sociólogo.Docente de la Universidad de Bs. As. y becario CONICET. 


\section{Referencias}

(1) HENITT; SMITH 1996 Y WHITE, "et al"El daño y la evolución del riesgo en América Central. Red de estudios Sociales en Prevención de Desastres en América Latina 1999. (1983); Costa Rica.

(2) LA RED. Red de Estudios Sociales en Prevención de desastres en América Latina. Desinventar; sistema de inventarios de Desastres en América Latina. OSSO / ITDG: Cali.

(3) COHEN, R. Salud Mental Para Víctimas De Desastres. Manual Para Trabajadores. Editorial El Manual Moderno. OPS. 1999. México. D.F.

(4) PICHOT, PIERRE, LÓPEZ-IBOR, "et al" 1995. D.S.M. IV Manual Diagnóstico y Estadístico de Trastornos Mentales. $4^{\circ}$ Edición. Editorial Masson, Barcelona.

(5) OPS- OMS- Los desastres naturales y la protección de la salud 1999. Asistencia humanística en casos de desastres. Washington. DC. 2000. Washington D.C.

(6) KIELHOFNER, G.: Modelo de la Ocupación Humana, teoría y aplicación, tercera edición. Investigación. Editorial: Panamericana . Buenos Aires. Argentina. 2002. Cap.27, pág.605.

(7) ROSENFELD, M.S. 1989. Ocupational disruption and adaptation: A study of house fire victims en American Journal of Ocupational Therapy 4, 89 -96.

(8) KIELHOFNER, G.: Modelo de la Ocupación Humana, teoría y aplicación, tercera edición. Investigación. Editorial: Panamericana . Buenos Aires. Argentina. 2002. Cap.27, pag.617.

(9) KIELHOFNER, G.: Modelo de la Ocupación Humana, teoría y aplicación, tercera edición. Investigación. Editorial: Panamericana . Buenos Aires. Argentina. 2002. Cap.27, pag.618.

(10) FAHER, R; PECCI, M; GÓMEZ PRIETO, C; BESONI, A Y GARZARÓN, M.. Salud Mental: Consecuencias Psicológicas de las explosiones ocurridas en la Fábrica Militar Río III, Córdoba, Argentina. En acta Psiquiátrica- Psicológica. 1997Editorial América Latina. 
(11) ALGARAÑAS, BADARIOTI Y BOSSIO: "Organización de la rutina de personas afectadas por el Mal de Alzheimer en el estadío I de la enfermedad que concurren al consultorio externo del Hospital J. B. Iturraspe de la ciudad de Santa Fe" Trabajo Final de la Licenciatura En Terapia Ocupacional. Escuela Superior de Sanidad Dr. Ramón Carrillo. Universidad Nacional del Litoral. Santa Fe. Argentina. 2003.

(12) YERXA, E. J,; et al. (1989). An Introduction to occupational science: A Fundation for occupational al therapy in the 21 century. Occupational therapy in Heald Care.

(13) CLARK , F. The concepts of habit and norifine: A preliminary Theoritical synthenis - Occupational Therapy Journal y Research, . (2000)20-1235-1375.

(14) POLKINGHOME, D.. Trasformative narratives: from victimc to agenetic life plots. American Journal of Occupational Therapy. (1996)Vol. 50(4).p.p.299305.

- Referencia y correspondencia de los autores "

Carla Boggio R.: Terapista Ocupacional. Maestrando en la Especialización y Maestría en Docencia Universitaria de la Universidad Nacional del Litoral (UNL). Profesora Titular Interina de la UNL. Miembro de la Comisión Evaluadora de Trabajos Finales de la Licenciatura en Terapia Ocupacional de la Universidad Nacional del Litoral.

Dirección Postal: Pasaje Paillet 1770. Esperanza. C.P.: 3080.

TE.: 03496-425992.

Dirección electrónica: crboggio@yahoo.com.ar

Daniela Chiapessoni: Terapista Ocupacional. Maestrando en la Especialización y Maestría en Docencia Universitaria de la Universidad Nacional del Litoral (UNL). Profesora Adjunta de la UNL.

Dirección Postal: Pasaje Ingenieros 5468. Santa Fe. C.P.: 3000.

TE: $0342-4883384$.

Dirección electrónica: danielachiapessoni@hotmail.com

Juan Carlos Funes: Licenciado en Psicología. Doctorando el Doctorado en Psicología de la Universidad de Buenos Aires (UBA). Profesor Jefe de Trabajos Prácticos semiexclusivo de la Universidad Nacional del Litoral.

Dirección Postal: Los Pinos s|n. Villa California. San José del Rincón. C.P.: 3000. TE.: 0342-4971686.

Dirección electrónica: jcfunes19@hotmail.com 
María del Valle Arenaza: Terapista Ocupacional. Maestrando en la Especialización y Maestría en Docencia Universitaria de la Universidad Nacional del Litoral (UNL).

Profesora Ayudante de cátedra la UNL.

Dirección Postal: Domingo Faustino Sarmiento .P.: 3000. Santa Fe.

TE: $0342-4563565$.

Dirección electrónica: malearebaza@hotmail.com

Mariana Boffelli: Terapista Ocupacional. Profesora Jefa de Trabajos Prácticos simple de la Universidad Nacional del Litoral. Coordinadora de la Licenciatura en Terapia Ocupacional de la Universidad Nacional del Litoral.

Dirección Postal: Laprida 4344.C.P. 3000. Santa Fe.

TE: 0342-4521671.

Dirección electrónica: boffelli@fbcb.unl.edu.ar

Mariquita Heit: Licenciada en Terapia Ocupacional. Pasante de Cátedra de la Universidad Nacional del Litoral.

Dirección Postal: Juan de Garay 4108. C.P. 3000. Santa Fe.

TE: $0342-4584637$.

Dirección electrónica: mariquitaheit@ciudad.com.ar

Mauro Demichelis: Licenciado en Terapia Ocupacional. Pasante extensionista de la Universidad Nacional del Litoral.

Dirección Postal: Alvear 3841. C.P. 3000. Santa Fe.

TE: 0342-4520911.

Dirección electrónica: $\underline{m}$ demichelis@hotmail.com

Milagros Demiryi: Terapista Ocupacional. Especialista en Docencia Universitaria. Maestrando en la Maestría en Docencia Universitaria de la Universidad Nacional del Litoral (UNL). Profesora Jefe de Trabajos Prácticos de la Universidad Nacional del Litoral. Profesora Adjunta en la Universidad Nacional de Quilmes. Miembro de la Comisión Evaluadora de Trabajos Finales de la Licenciatura en Terapia Ocupacional de la Universidad Nacional del Litoral.

Dirección Postal: San Juan 2438. C.P. 3000. Santa Fe.

TE: 0342-4811354.

Dirección electrónica: avenida@arnet.com 